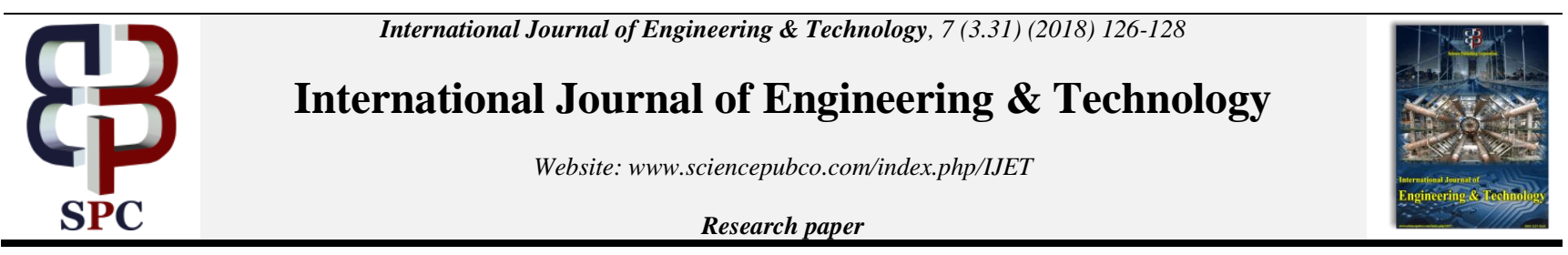

\title{
Generalized Iterated Function Systems Containing Functions of Integral Type
}

\author{
Minirani $\mathbf{S}^{1 *}$ \\ ${ }^{I}$ Dept. of BSH, MPSTME, SVKM's NMIMS Deemed to be University \\ *Corresponding AuthorE-mail: miniranis@yahoo.com
}

\begin{abstract}
A finite collection of mappings which are contractions on a complete metric space constitutes an iterated function system. In this paper we study the generalized iterated function system which contain generalized contractions of integral type from the product space $M^{m} \rightarrow M$. We prove the existence and uniqueness of the fixed point of such an iterated function system which is also known as its attractor.
\end{abstract}

Keywords: attractor; fixed point; generalized iterated function system; integral type contractions; product space.

\section{Introduction}

J. Hutchinson [4] introduced the iterated function system in the present form, which is a finite collection of mappings which are contractions on a complete metric space, but it was popularized in the research area by M. Barnsley [7]. Much of a research has been carried out to extend this classical framework of fractals to general and infinite spaces. Results regarding the infinite IFS and generalized IFS can be found in the works by N. A. Secelean [12], D. Dumitru [3], R. Miculescu and A. Mihail [10, 11]. An iterated function system is a simple mathematical tool for constructing a fractal set through a finite collection of contraction mappings. Since the IFS help to obtain a good approximation of objects in the real world, it serves as a basis for some data compression methods.

Here, our aim is to extend the contraction $f: M \rightarrow M$ of integral type to functions $f: M^{m} \rightarrow M$ and use it to define generalized integral type Iterated Function System (GI-IFS) and study the fixed point of such a GI-IFS.

\section{Iterated Function Systems}

Let us discuss some of the well-known results required to understand iterated function system. Throughout the paper, $(M, d)$ will denote a complete metric space and $C(M)$ will denote the set of all non-empty compact subsets of $M$.

Definition 2.1 [5]: 'The Hausdorff metric $d_{H}$ on $C(M)$ is defined by $d_{H}(A, B)=\max \left\{\sup _{x \in A}\{\operatorname{dist}(x, B)\}, \sup _{y \in B}\{\operatorname{dist}(y, A)\}\right\}$

for $A, B \in C(M)$ '.

We can see that $C(M)$ is complete in the metric $d_{H}$. That is, if $x, y \in M$ and $A, B \in C(M)$, we have the distance from a point $x$ to the set $\mathrm{B}$ defined as $d(x, B)=\min \{d(x, y) ; y \in B\}$ and the distance from set $A$ to set $B$ defined as $d(A, B)=\max \{d(x, B) ; x \in A\}$. Then the Hausdorff distance from a set $A$ to a set $B$ is defined as $d_{H}(A, B)=d(A, B) \vee d(B, A)$. Defining so, $d_{H}$ is a metric defined on $C(M)$.

Definition 2.2 [5]: A mapping $w: M \rightarrow M$ on the metric space $(M, d)$ is said to be a contraction if there is a number $s$ with $0<s<1$ such that $d(w(x), w(y)) \leq s d(x, y), \forall x, y \in M$. We can easily prove that such a mapping is continuous. If it becomes an equality, ie., if $d(w(x), w(y))=s d(x, y)$, then $w$ is called a similarity as it transforms sets into geometrically similar ones.

In 1922, a Polish mathematician S. Banach [13] proved one of the very important result which paved the way to fixed point theory which is known as the Banach contraction principle.

Theorem 2.3: Let $w: M \rightarrow M$ be a contraction mapping on a complete metric space $(M, d)$ with the contractivity factor ' $s$ '. Then $w$ possesses exactly one fixed point $x^{a} \in M$. Moreover, for any point $x \in M$, the sequence $\left\{w^{n}(x) ; n=0,1,2, \ldots\right\}$ converges to $x^{\natural}$. ie., $\lim w^{n}(x)=x^{a}$, for each $x \in M$.

The theorem due to Banach stated above guarantees the existence and uniqueness of the fixed point of an IFS which consists of a set of contractive maps over a complete metric space.

Definition 2.4: A complete metric space $(M, d)$ together with a finite set of contraction mappings $w_{k}: M \rightarrow M$ with respect to the contraction factors $s_{k}$, for $k=1,2, \ldots, N$ constitutes a (hyperbolic) Iterated Function System [6]. The abbreviation IFS is 
used for iterated function system. The notation of the IFS just defined is $\left\{M ; w_{k}, \mathrm{k}=1,2, \ldots, N\right\}$ and its contraction factor is $s=\max \left\{s_{k}: \mathrm{k}=1,2, \ldots, N\right\}$. The IFS is hyperbolic since each $w_{k}$ in the definition is a contraction mapping.

Theorem 2.5: [6] Let $\left\{M ; w_{k}, 1 \leq \mathrm{k} \leq N\right\}$ be a hyperbolic IFS with contraction factor $s$. Then the transformation $W: C(M) \rightarrow C(M) \quad$ defined by $W(B)=\bigcup_{k=1}^{N} w_{k}(B)$ for all $B \in C(M)$, is a contraction mapping on the complete metric space $(C(M), h)$ with contraction factor $s$. That is, $d_{H}(W(B), W(C)) \leq s . h(B, C)$ for all $B, C \in C(M)$. Its unique fixed point, $A \in C(M)$, obeys $A=W(A)=\bigcup_{k=1}^{N} w_{k}(A)$ and is given by $A=\lim _{n \rightarrow \infty} W^{\circ n}(B)$ for any $B \in C(M)$, where $W^{\circ n}$ denotes $W o$......o $W$ (n times).

The fixed point $A \in C(M)$ described in the above theorem is called the attractor of the IFS.

Every IFS can be considered as a particular case of generalized iterated function system (GIFS).

Definition 2.6: Let $\left(M^{m}, f_{i}: 1 \leq i \leq n\right)$ be a GIFS. The function $F: C\left(M^{m}\right) \rightarrow C(M) \quad$ defined by $F\left(C_{1}, C_{2}, \ldots, C_{m}\right)=\bigcup_{i=1}^{n} f_{i}\left(C_{1} \times C_{2} \times \ldots \times C_{m}\right)$ is called the fractal operator associated with the GIFS [3]. The function $F$ is a contraction with contraction factor $c \leq \max _{i=1, \ldots, n} c_{i}$ where $c_{i}$ is the contraction factor of $f_{i}$ for each $i$.

\section{Main Results}

In the classical definition of IFS, there is a finite collection of Banach contraction mappings on $M$. In this section we discuss about an IFS where each of the contractions are of integral type [8]. The following result by A. Branciari [1] was the motivation behind the definition of integral type IFS.

Theorem 3.1: Let $(M, d)$ be a complete metric space, $s \in(0,1)$, and let $\mathrm{g}: M \rightarrow M$ be a mapping such that for each $x, y \in M$,

$$
\int_{0}^{d(\mathrm{~g} x, \mathrm{~g} y)} \phi(t) d t \leq s \int_{0}^{d(x, y)} \phi(t) d t,
$$

where $\phi:[0, \infty) \rightarrow[0, \infty)$ is a Lebegue -integrable mapping on each compact subset of $[0, \infty)$, nonnegative, and such that for each ò $>0, \int_{0}^{\grave{b}} \phi(t) d t>0$; then f has a unique fixed point $a \in M$ such that for each $x \in M, \lim _{n \rightarrow \infty} g^{n}(x)=a$.

We have defined the Integral type IFS $[8,9]$ on the basis of definition of hyperbolic iterated function system given by Barnsley [7].

Definition 3.2: An integral type-iterated function system (I-IFS) consists of a complete metric space $(M, d)$ together with a finite set of integral type-contraction mappings $f_{n}: M \rightarrow M$ having contraction factors $k_{n} \in(0,1)$, for $n=1,2,3, \ldots, N$. We name $k_{n}$ as the I-contractivity factor of integral type contraction $f_{n}$, for each $n=1,2,3, \ldots, N$.

The proof of the following theorem can be found in [8].

Theorem 3.3: Let $(M, d)$ be a complete metric space and $\left\{M ; f_{n}: 1 \leq n \leq N\right\}$ be an I-IFS having I-contractvity factors $k_{n}$ or each $n$. Consider the transformation $F: C(M) \rightarrow C(M)$ given by $F(B)=\bigcup_{n=1}^{N} f_{n}(B)$ for each $B \in C(M)$. Then $F$ is a contraction on the complete metric space $\left(C(M), d_{H}\right)$ with Icontractivity factor $k=\max \left\{k_{n}: 1 \leq n \leq N\right\}$. Its unique fixed point, $A \in C(M)$, obeys $A=F(A)=\bigcup_{n=1}^{N} f_{n}(A)$ and is given by $A=\lim _{n \rightarrow \infty} F^{o n}(B)$ for any $B \in C(M)$.

Here $A \in C(M)$ as defined above becomes the attractor of the IIFS $\left\{M ; f_{n}: 1 \leq n \leq N\right\}$.

We now introduce the notion of integral type conditions for functions $f: M^{m} \rightarrow M$. On the product space we consider the metric $d\left(\left(\mathrm{p}_{1}, \ldots p_{m}\right),\left(\mathrm{q}_{1}, \ldots \ldots, \mathrm{q}_{m}\right)\right)=\max .\left\{d\left(\mathrm{p}_{1}, \mathrm{q}_{1}\right), \ldots, d\left(\mathrm{p}_{m}, \mathrm{q}_{m}\right)\right\}$.

Let $(M, d)$ be a metric space. and $f: M^{m} \rightarrow M$ where $m \in \square$.

We say that $\mathrm{f}$ is satisfying the integral type condition if for $c \in(0,1)$ and for each $p=\left(\mathrm{p}_{1}, \ldots \ldots, \mathrm{p}_{m}\right), \mathrm{q}=\left(\mathrm{q}_{1}, \ldots \ldots, \mathrm{q}_{m}\right) \in X^{m}$, $\int_{0}^{d\left(f\left(\mathrm{p}_{1}, \ldots, \mathrm{p}_{m}\right), f\left(\mathrm{q}_{1}, \ldots, \mathrm{q}_{m}\right)\right)} \phi(t) d t \leq c_{i} \int_{0}^{d\left(\mathrm{p}_{i}, \mathrm{q}_{i}\right)} \phi(t) d t$,

for each i: $1 \leq i \leq m$.

Theorem 3.4: Let $(M, d)$ be a complete metric space. For $m \in \square$ let $f: M^{m} \rightarrow M$ be a function of integral type. Then $\mathrm{f}$ has a unique fixed point.

Proof: For $m=1$, this becomes the well known result which has been proved by A. Biancari [1], that is, there exist a unique fixed point for an integral type contraction $f: M \rightarrow M$.

So here we consider the case where $m>1$. Let us define $g: M \rightarrow M$ as $g(\mathrm{p}):=f(\mathrm{p}, \mathrm{p}, \ldots, \mathrm{p})$, for all $p \in M$. Since $f: M^{m} \rightarrow M$ is a function satisfying integral type condition, by definition, for $c \in(0,1)$ and for each $p=\left(\mathrm{p}_{1}, \ldots \ldots, \mathrm{p}_{m}\right), \mathrm{q}=\left(\mathrm{q}_{1}, \ldots \ldots, \mathrm{q}_{m}\right) \in M^{m}$, $\int_{0}^{d\left(f\left(\mathrm{p}_{1}, \ldots, \mathrm{p}_{m}\right), f\left(\mathrm{q}_{1}, \ldots, \mathrm{q}_{m}\right)\right)} \phi(t) d t \leq c_{i} \int_{0}^{d\left(\mathrm{p}_{i}, \mathrm{q}_{i}\right)} \phi(t) d t$, for each $i: 1 \leq i \leq m$. Let us take $p_{1}=p_{2}=\ldots=p_{m}=p$ and $q_{1}=q_{2}=\ldots=q_{m}=q$ with $\mathrm{p}, \mathrm{q} \in M$, and $\int_{0}^{d(f \mathrm{p}, \mathrm{fq})} \phi(t) d t \leq c \int_{0}^{d(\mathrm{p}, \mathrm{q})} \phi(t) d t$, then we have $\int_{0}^{d(f(\mathrm{p}, \mathrm{p}, \ldots, \mathrm{p}, f(\mathrm{q}, \mathrm{q}, \ldots, \mathrm{q}))} \phi(t) d t \leq c \int_{0}^{d(\mathrm{p}, \mathrm{q})} \phi(t) d t$

Thus we have for each $x, y \in M, \quad \int_{0}^{d(g(\mathrm{p}) \cdot g(\mathrm{q}))} \phi(t) d t \leq c \int_{0}^{d(\mathrm{p}, \mathrm{q})} \phi(t) d t$, Thus $g$ is a function of integral type condition. This implies that there exist an $a \in M$ such that $g(a, a, \ldots, a)=a$. Let $a, b \in M$ 
such that $a \neq b$ be two fixed points of $f$. Then

$f(a, a, \ldots, a)=a$ and $f(b, b, \ldots, b)=b$ which means

$g(a)=a$ and $g(b)=b$. Since $g$ is an integral type mapping on a complete metric space it has a unique fixed point and so $a=b$.

Theorem 3.5: Let $f_{i}: M^{m} \rightarrow M$ be an integral type contraction mapping on a complete metric space $(M, d)$ with I-contractivity factor ' $k$ '. Then the operator $F: C(M)^{m} \rightarrow C(M)$ defined by $F\left(B_{1}, \ldots, B_{m}\right)=\bigcup_{i=1}^{m} f_{i}\left(B_{1} \times B_{2} \times \ldots \times B_{m}\right)$ is an integral type contraction having a unique fixed point such that $F(A, A, \ldots, A)=A$.

Proof: Since each $f$ is a contraction of integral type, we have $\int_{0}^{d\left(f_{i}\left(x_{1}, \ldots, x_{m}\right), f_{i}\left(y_{1}, \ldots, y_{m}\right)\right)} \phi(t) d t \leq c_{j} \int_{0}^{d\left(x_{j}, y_{j}\right)} \phi(t) d t$, for each $x_{j}, y_{j} \in X$ where $j \in\{1,2, \ldots, m\}$. Let us choose $A_{j}, B_{j} \in C(M)$. We will prove that $\int_{0}^{d_{H}\left(F\left(A_{1}, \ldots, A_{m}\right), F\left(B_{1}, \ldots, B_{m}\right)\right)} \phi(t) d t \leq c_{j} \int_{0}^{d_{H}\left(A_{j}, B_{j}\right)} \phi(t) d t$.

To prove this, let us choose an arbitrary $z \in F\left(A_{1}, \ldots, A_{m}\right)$. Then there exists an $i \in\{1,2, \ldots, m\}$ and $x_{1} \in A_{1}, \ldots, x_{m} \in A_{m}$ such that $z=f_{i}\left(x_{1}, \ldots, x_{m}\right)$.

By the definition of Hausdorff metric there exists a $y_{1} \in B_{1}, \ldots, y_{m} \in B_{m}$ such that $\int_{0}^{d\left(x_{j}, y_{j}\right)} \phi(t) d t \leq c_{j} \int_{0}^{d_{H}\left(A_{j}, B_{j}\right)} \phi(t) d t$. This implies that $\int_{0}^{d\left(f_{i}\left(x_{1}, \ldots, x_{m}\right), f_{i}\left(y_{1}, \ldots, y_{m}\right)\right)} \phi(t) d t \leq c_{j} \int_{0}^{d_{H}\left(A_{j}, B_{j}\right)} \phi(t) d t$. Then we have $\inf \quad d\left(f_{i}\left(x_{1}, \ldots, x_{m}\right), f_{i}\left(w_{1}, \ldots, w_{m}\right)\right)=d\left(z, f_{i}\left(B_{1}, \ldots, B_{m}\right)\right)$ and so $\int_{0}^{d\left(z, f_{i}\left(B_{1}, \ldots, B_{m}\right)\right)} \phi(t) d t \leq c_{j} \int_{0}^{d_{H}\left(A_{j}, B_{j}\right)} \phi(t) d t$. Hence we have
\[ \int_{0}^{d\left(z, F\left(B_{1}, \ldots, B_{m}\right)\right)} \phi(t) d t \leq c_{j} \int_{0}^{d_{H}\left(A_{j}, B_{j}\right)} \phi(t) d t . \]

As $z$ is arbitrarily chosen from $F\left(A_{1}, \ldots, A_{m}\right)$, and since $F\left(A_{1}, \ldots, A_{m}\right)$ is a compact set in $C(M)^{m}$ and the function $z \rightarrow d\left(z, F\left(B_{1}, \ldots, B_{m}\right)\right) \quad$ continuous, we have $d\left(F\left(A_{1}, \ldots, A_{m}\right), F\left(B_{1}, \ldots, B_{m}\right)\right)=$ sup $d\left(z, F\left(B_{1}, \ldots, B_{m}\right)\right)$ So we have $\int_{0}^{d\left(F\left(A_{1}, \ldots, A_{m}\right), F\left(B_{1}, \ldots, B_{m}\right)\right)} \phi(t) d t \leq c_{j} \int_{0}^{d_{H}\left(A_{j}, B_{j}\right)} \phi(t) d t \quad$ Proceeding in the similar way we can prove that $\$ \int_{0}^{d\left(F\left(B_{1}, \ldots, B_{m}\right), F\left(A_{1}, \ldots, A_{m}\right)\right)} \phi(t) d t \leq c_{j} \int_{0}^{d_{H}\left(A_{j}, B_{j}\right)} \phi(t) d t$. This completes our proof and so $F\left(B_{1}, \ldots, B_{m}\right)=\bigcup_{i=1}^{m} f_{i}\left(B_{1} \times B_{2} \times \ldots \times B_{m}\right)$ is an integral type contraction. So by the theorems above, there exists a unique element $A \in M$ such that $F(A, A, \ldots, A)=A$ and this fixed point of $F$ is called the attractor of the generalized I-IFS defined on $C(M)^{m}$.

Example 3.6: Let $M=\square^{+}$with the Euclidean metric $d=|$.$| ,$ $\phi: \square^{+} \rightarrow \square^{+}$and $f: M \rightarrow M$ defined as follows: $\phi(t)=1 \forall t \in \square^{+}$and $f(x)= \begin{cases}0 & x \in[0,1] \\ \frac{x}{2} & x \in(1, \infty)\end{cases}$

If $x, y \in[0,1], \int_{0}^{d(f x, f y)} \phi(t) d t=0 \leq c \int_{0}^{d(x, y)} \phi(t) d t$.

If $x, y \in(1, \infty)$, then

$\int_{0}^{d(f x, f y)} \phi(t) d t=\frac{|x-y|}{2} \leq \frac{1}{2}|x-y| \leq \frac{1}{2} \int_{0}^{d(x, y)} \phi(t) d t$. Hence the conditions are satisfied and $f$ has a unique fixed point according to the theorem.

\section{Conclusion}

In this paper we have generalized the contraction mapping which is of integral type to the product space. Using such contractions, we have defined a generalised IFS and studied its fixed point on a product space which is complete. An example to support the fixed point theorem of integral type is also given for completion. The topological properties of integral type IFS will be carried out in the upcoming research papers.

\section{Acknowledgement}

The author would like to extend extreme gratitude to the anonymous reviewers for their helpful comments and suggestions which has contributed to the improvement of the paper.

\section{References}

[1] A. Branciari (2002), A fixed point theorem for mappings satisfying a general contractive condition of integral type, Int. J. Math. Math. Sci., 29(9), pp. 531-536.

[2] B. B. Mandelbrot, The Fractal Geometry of Nature, W. H. Freeman, (1982).

[3] D. Dumitru (2009), Generalised iterated function systems containing Meir-Keeler functions, An. Univ. Bucuresti. math. LVIII, pp. 3-15.

[4] J. E. Hutchinson (1981), Fractals and self similarity, Indiana Univ. Math. J. 30, pp. 713-747.

[5] K. J. Falconer, Fractal Geometry - Mathematical foundations and applications, John Wiley Sons, (1990).

[6] K. J. Falconer, The Geometry of Fractal Sets, Cambridge University Press, (1985).

[7] M. F. Barnsley, Fractals Everywhere, Academic Press, Boston, MA, (1988).

[8] S. Minirani (2017), Fixed points of iterated function systems of integral type, (Under review).

[9] S. Minirani (2018), Fixed point of iterate function system containing Meir Keeler integral type contractions, Mathematical Sciences International Research Journal, 7 (1), pp. 391-395.

[10] R. Miculescu and A. Mihail (2016), Reich-type iterated function systems, J. Fixed Point Theory Appl., 18, pp. 285296.

[11] R. Miculescu and A. Mihail (2008), R. Vrscay, Applications of fixed point theorems in the theory of generalized IFS, Fixed Point Theory Appl., 2, article ID 312876.

[12] N. A. Secelean (2001), Countable iterated function systems, Far East J. Dyn. Syst., 3, pp. 149-167.

[13] S. Banach (1922), Sur les operations dans les ensembles abstrait et leur application aux equations, integrals, Fundam. math., 3, pp. 133-181. 